\section{Update zur Kombination BRAF- plus MEK- Inhibition bei fortgeschrittenem Melanom}

\author{
In der coBRIM-Studie war die Kombination aus MEK-Hemmer und BRAF- \\ Inhibitor dem BRAF-Hemmer alleine beim progressionsfreien Überleben (PFS) \\ überlegen. Nun wurden die Ergebnisse aktualisiert.
}

$\mathrm{n}$ die doppelblinde, randomisierte, placebokontrollierte Studie wurden 495 Patienten mit BRAF-V600-mutiertem Melanom im Stadium IIIC oder IV aufgenommen. Sie erhielten entweder den

MEK-Inhibitor Cobimetinib (pro Zyklus $60 \mathrm{mg} / \mathrm{d}$ für 21 Tage, dann 7 Tage Pause) oder Placebo in Kombination mit dem BRAF-Inhibitor Vemurafenib (2-mal 960 $\mathrm{mg} / \mathrm{d}$ ). Primärer Endpunkt war das PFS.

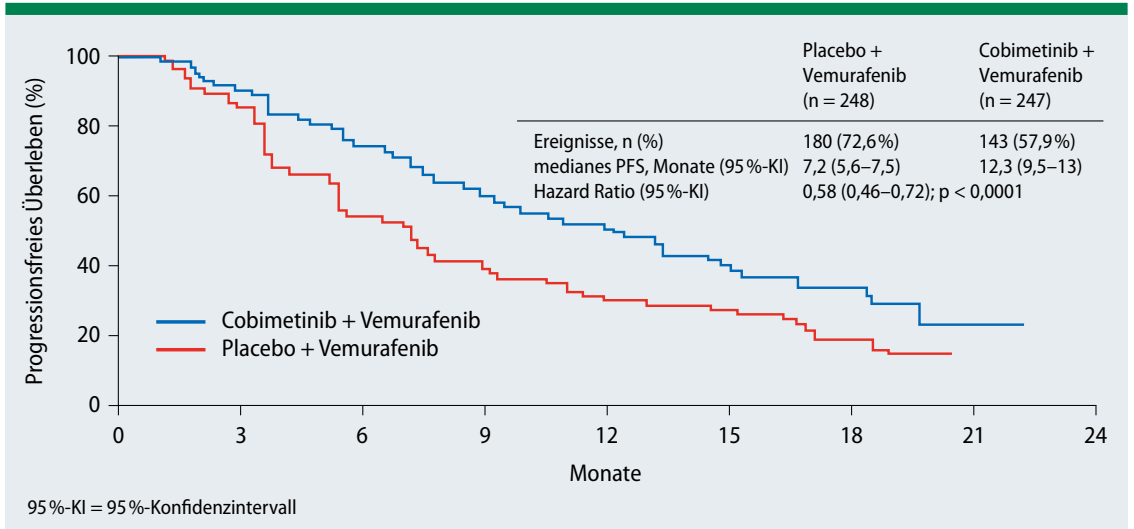

Abb. 1: Die Patienten unter der Kombinationstherapie leben länger progressionsfrei
Nach median 14,2 Monaten war die Kombination beim medianen PFS überlegen (12,3 vs. 7,2 Monate; Hazard Ratio [HR] 0,58; 95\%-Konfidenzintervall [95\%-KI] 0,46-0,72; p < 0,0001) (Abb. 1), ebenso in der finalen Analyse des Gesamtüberlebens (22,3 vs. 17,4 Monate; HR 0,70, $95 \%$-KI 0,55-0,90; $\mathrm{p}=0,005)$.

Zudem erwies sich die Kombination als verträglich, mit akzeptablem Sicherheitsprofil. Häufigste Grad 3/4-Nebenwirkungen, die unter der Kombination öfter auftraten als unter Vemurafenib allein, waren Anstiege der $\gamma$-GT (15 vs. $10 \%$ ), der Kreatinphosphokinase (12 vs. $<1 \%$ ) und der ALT (11 vs. 6\%). Schwerwiegende unerwünschte Ereignisse traten bei $37 \%$ der Patienten unter der Kombination und bei 28\% unter der Monotherapie auf.

Fazit: Diese Ergebnisse bestätigen den klinischen Nutzen der Kombination aus Cobimetinib plus Vemurafenib. Sie wurde als Erstlinientherapie beim fortgeschrittenen Melanom mit BRAF-V600Mutation zugelassen. Judith Neumaier

Ascierto PA et al. Cobimetinib combined with vemurafenib in advanced BRAF(V600)-mutant melanoma (coBRIM): updated efficacy results from a randomised, double-blind, phase 3 trial. Lancet Oncol. 2016;17(9):1248-60.

\title{
Metastasiertes Melanom: PD1-Inhibitor verbessert auch die Lebensqualität
}

\section{Der PD1-Inhibitor Nivolumab führte in der Phase-III-Studie CheckMate 066 bei therapienaiven Patienten mit metastasiertem Melanom zu einem signifi- kanten Überlebensvorteil im Vergleich zur Chemotherapie mit Dacarbazin. Nun wurden Daten zur gesundheitsbezogenen Lebensqualität präsentiert.}

n der Studie erhielten 418 therapienaive Patienten mit metastasiertem Melanom Nivolumab oder Dacarbazin. Primärer Endpunkt war das Gesamtüberleben. Die gesundheitsbezogene Lebensqualität (QoL) wurde bei Studienbeginn und danach alle 6 Wochen mit den Fragebögen QLQ-C30 der European Organisation for Research and Treatment of Cancer (EORTC) und EuroQoL Five Dimensions Questionnaire (EQ-5D) erhoben. Die mittleren Scores waren zu Studienbeginn in beiden Gruppen vergleichbar. Unter Nivolumab blieb die gesundheits- bezogene QoL über die gesamte Studiendauer erhalten. In beiden Gruppen ergab sich bis Woche 7 eine mäßige, aber nicht signifikante und klinisch nicht relevante Verbesserung der Scores für die globale Lebensqualität. In Woche 25 zeigte sich unter Dacarbazin, nicht aber unter Nivolumab, ein Trend zu einer Verschlechterung. Bei Patienten unter Nivolumab verschlechterten sich einige EORTCQLQ-C30-Skalen und der „EQ-5D utility index" signifikant später.

Die explorative Analyse zeigte unter Nivolumab bessere EQ-5D-Scores als unter Dacarbazin. Zudem ergaben sich unter Nivolumab klinisch relevante Verbesserungen im EQ-5D in den Wochen 37, 61 und 67. Unter Nivolumab war in den EORTC QLQ-C30-Skalen kein Anstieg der Symptomlast zu beobachten.

Fazit: Diese Daten zeigen, dass Nivolumab bei Patienten mit fortgeschrittenem Melanom das Überleben im Vergleich zu Dacarbazin verlängert und zum Erhalt der gesundheitsbezogenen QoL beiträgt. Insgesamt ist damit das NutzenRisiko-Profil von Nivolumab dem von Dacarbazin überlegen. Judith Neumaier

Long GV et al. Effect of nivolumab on health-related quality of life in patients with treatment-naïve advanced melanoma: results from the phase III CheckMate 066 study. Ann Oncol. 2016;27(10):1940-6. 